\title{
Distance, structure and bright stellar content of the dwarf irregular galaxy UGC 685
}

\author{
U. Hopp ${ }^{1, \star}$ \\ Universitätssternwarte München, Scheiner Str. 1, D-81679 München, Germany, \\ e-mail: hopp.at.usm.uni-muenchen.de
}

Received June 16; accepted August 27, 1998

\begin{abstract}
B$ and $R$ CCD images and $J$ NICMOS3 frames taken with the Calar Alto $3.5 \mathrm{~m}$ telescope of the dwarf irregular galaxy UGC 685 are presented. The brightest part of the stellar population is resolved in $B$ and $R$, very few also in $J$. The stellar color-magnitude diagram is discussed. An estimate of the distance to UGC 685 of $5.5 \mathrm{Mpc}$ is derived based on the brightest blue supergiant stars. Most of the resolved bright stars show colors in the range $-0.1 \leq B-R \leq+0.7$.
\end{abstract}

The continuum light of the unresolved stars is distributed rather regular in $B, R$, and $J$, showing only minor irregularities. This surface brightness distribution follows an exponential law in all three colors with a scale length $r_{\mathrm{c}}=0.33 \mathrm{kpc}$. The central surface brightnesses are $21.57 \pm 0.09 \mathrm{mag} / \square^{\prime \prime}, 20.65 \pm 0.06 \mathrm{mag} / \square^{\prime \prime}$, and $20.11 \pm$ $0.11 \mathrm{mag} / \square^{\prime \prime}$, in $B, R$, and $J$, respectively. The surface brightness can be traced out to $5 r_{\mathrm{c}}$ in $B$ and $R$. Thus, UGC 685 belongs to the class of dwarfs where the HI distribution is much more extended (here 2.6 times) than the optical (stellar) light distribution, but contrary to many objects of this type, it does not belong to the class of lowsurface brightness objects. No color gradient was detected in UGC 685 except that the very center is slightly bluer. The overall colors are $B-R=+0.97, B-J=+1.55$ and the magnitude is $B=14.55\left(M_{B}^{0}=-14.5\right)$.

The classification as an irregular dwarf from survey plates results from the few HII regions of UGC 685 which are all concentrated to the South-East of the center of the galaxy. On an $\mathrm{H} \alpha$ Calar Alto $2.2 \mathrm{~m}$ telescope CCD image, I identified only 5 HII regions, one of them being rather bright. The total $\mathrm{H} \alpha$ flux corresponds to a mildly on-going star formation with a rate of $0.003 M_{\odot} \mathrm{yr}^{-1}$, a low rate even in comparison to other dwarfs. The available (and

Send offprint requests to: U. Hopp

* Visiting Astronomer, German-Spanish Astronomical Center, Calar Alto, operated by the Max-Planck-Institut für Astronomie jointly with the Spanish National Commission for Astronomy. limited) data do not indicate any major deviation from this rather low star formation rate within the last $10^{9} \mathrm{yr}$.

Key words: galaxies — dwarfs; galaxies — structure; galaxies - distances

\section{Introduction}

Isolated dwarf irregular galaxies are good laboratories to study the star formation history as well as the evolution of galaxies in the absence of external triggers and without the influence of large-scale internal triggers like density waves (see Hunter 1997, for a review). Understanding local dwarfs constrains the models which were developed for a description of the large population of sub-luminous, irregular systems at medium redshifts (see Babul \& Ferguson 1996; Ferguson \& Babul 1998). Very nearby galaxies of this type are best suited for detailed analysis of their star formation histories as they can be resolved into individual stars (see e.g. Gallart et al. 1996, their Table 1, and Greggio 1994 and references in these papers). Inside the Local Group (distance up to roughly $1 \mathrm{Mpc}$ ), groundbased deep images under excellent seeing conditions can be used for this type of investigation while for more distant galaxies (up to about roughly $5-10 \mathrm{Mpc}$ ), HST data are essential (e.g. Schulte-Ladbeck et al. 1998). For galaxies in this distance range, radial velocities are very crude distance estimators as the peculiar velocities are in the order of or larger than the Hubble flow. A quick and inexpensive method to reject more distant interlopers from the $10 \mathrm{Mpc}$ sample is the identification of the brightest stars which can be done with ground-based images of good seeing (see the discussion by Karachentsev \& Tikhonov 1993, K\&T hereafter), and Rozanski \& Rowen-Robinson 1994, R\&RR). Further, the ground based images indicate the regions of recent high star formation activities (HII regions, star clusters), thus allowing a pre-selection for the 
pointing of the HST or adaptive optic observations with their rather small field of view.

UGC 685 (=CGCG0104.7+1625), which belongs to the 10 Mpc sample of Kran-Korteweg \& Tammann (1979), was classified as a late type dwarf galaxy (Sm). KranKorteweg \& Tammann could not associate UGC 685 to one of their groups and therefore called it a field galaxy. This indicates that at least the recent evolution and star formation history of UGC 685 was free of important triggers by interaction, albeit small mass HI companions as found in several cases by Taylor et al. (1996) can not be ruled out. The available optical data as recently compiled by Schmidt \& Boller (1992a,b) indicate a rather normal and absolutely faint dwarf galaxy at a systemic velocity (corrected for the local group flow) of $377 \mathrm{~km} \mathrm{~s}^{-1}$. The galaxy was included in various HI surveys which indicate an amount of $8.510^{7} M_{\odot}$ in neutral gas and a ratio of neutral gas to blue luminosity of 0.6 (Schmidt \& Boller 1992a,b), also rather normal for its type. As this dwarf galaxy appears relatively regular on survey plates and was indicated to be nearby, I selected it for a kinematic study of its stars and its ionized gas as well as for a study of its chemical abundances through optical long slit spectroscopy.

Only a radial velocity exists as distance indicator for UGC 685. Surface photometry which is necessary for the analysis of kinematic data is still missing. Finally, no colors or other values which hint at the star formation history, are published yet. I therefore included this dwarf galaxy in photometric CCD observations with the Calar Alto $3.5 \mathrm{~m}$ and $2.2 \mathrm{~m}$ telescopes to obtain multi-color surface photometry of the galaxy, its structural parameters and HII morphology and to resolve the brightest supergiants. These can yield an independent distance estimate, following the recent update of the calibration for the brightest blue supergiants by K\&T and R\&RR. The limitation and error budget of this techniques has been discussed in detail by R\&RR.

The observations and their reductions are described in Sect. 2. The structure and HII morphology, the colormagnitude diagram of the resolved stars and the distance estimate are presented in Sect. 3 and I conclude in Sect. 4.

\section{Observations and reductions}

\section{1. $B$ and $R$ band observations}

Prime focus CCD images were obtained with the Calar Alto $3.5 \mathrm{~m}$ telescope during a photometric night, the details are given in Table 1. After debiasing and flatfielding in the usual manner, I constructed an image which contains only the underlying smooth light distribution of the unresolved fainter stars as described by Hopp \& Schulte-Ladbeck (1991). This "smooth mask" was used to derive the structural parameters by applying the ellipticity fit of Bender \& Möllenhoff (1987). Then, the "smooth masks" were subtracted from the original $B$ and $R$ images which yielded frames with the resolved stars only. To these frames, DAOPHOT in its MIDAS version was applied, again following Hopp \& Schulte-Ladbeck (1991). The frames of both colors were searched independently by DAOPHOT and only those objects were accepted, which were found in both frames. Figure 1 shows the $B$ image of UGC 685.

\section{2. $H \alpha$ observations}

The Calar Alto $2.2 \mathrm{~m}$ telescope and its CCD camera were used to obtain $\mathrm{H} \alpha$ - and $R$-images of UGC 685 . The night was of poor photometric quality, thus only a preliminary calibration through the $R$ band observations was applied. Details are given in Table 1. As above, the usual CCD calibration frames were obtained and applied and the individual exposures in each filter were added. The $R$ image was flux-scaled to the $\mathrm{H} \alpha$-image with the aid of several medium-bright stars and then subtracted, yielding a continuum free $\mathrm{H} \alpha$ frame of UGC 685 (for more technical details see Bomans et al. 1997). Figure 2 shows the $\mathrm{H} \alpha$ image of UGC 685 .

\section{3. $J$ band observations}

$J$-band observations were taken with the Calar Alto $3.5 \mathrm{~m}$ telescope and its IR camera MAGIC during a photometric night. The set-up details can be found in Table 1. After every five object frames, nearby night sky data were taken at 4 different positions around UGC 685. The sets of object frames were slightly shifted against each other. The individual $10 \mathrm{~s}$. exposures were corrected for bias, dark current, night sky pattern and fixed-pattern noise with the usual calibration data. Then they were transformed into a common reference frame and finally added.

As the on-line facility did not show any signal of UGC 685 , no $\mathrm{K}$ data were obtained. For the same reason, centering was not perfect and the eastern outskirt of UGC 685 was therefore not mapped within the tiny field of view available with this set-up.

\section{Results and discussion}

\subsection{Morphology and total fluxes}

The $B$ (Fig. 1), $R$, and $J$ images show a mostly regular galaxy of elliptical shape. Only few brighter and extended knots in a region to the South-East of the center can be seen. The $\mathrm{H} \alpha$ (Fig. 2) image reveals that these knots are HII regions. To the limits of the images, one bright and 4 fainter, smaller HII regions were identified. The largest one has a diameter of about $9.3^{\prime \prime}(0.25 \mathrm{kpc})$. These few HII regions are clumped in an area of no more than $25^{\prime \prime}$ 
Table 1. Details of the observations presented in this paper

\begin{tabular}{l|c|c|c}
\hline observations & $B, R_{(\mathrm{c})}$ & $\mathrm{H} \alpha, R$ & $J$ \\
\hline date & Oct. 12, 1994 & Oct. 04, 1994 & Sep. 06, 1995 \\
telescope & CA 3.5 m Prim & CA 2.2 m Cas & 3.5 m Cas \\
detector & Tektronix CCD & Tektronix CCD & NICMOS3 HgCdTe \\
pixels & $1024 \times 1024$ & $1024 \times 1024$ & $256 \times 256$ \\
pixel size ["] & 0.53 & 0.28 & 0.33 \\
field of view ['] & 7 & 4.7 & 1.4 \\
& $($ circle) & (square) & (square) \\
total exp. time [sec] & 360,240 & 900,680 & 1200 \\
seeing [FWHM $\left.{ }^{\prime \prime}\right]$ & $1.1,1.3$ & $1.7,1.7$ & 1.5 \\
standard stars & NGC 2419, NGC 7790 & - & Elias et al. \\
& Christian et al. 1985 & & 1982 \\
\hline
\end{tabular}

Table 2. Overall properties of UGC 685 from the literature and as derived in this paper. Absolute magnitudes are corrected for galactic reddening with the given extinction value from Burstein \& Heiles (1982), distance depend values are converted with the new distance estimate of $5.5 \mathrm{Mpc}$

\begin{tabular}{l|c|l}
\hline property & value & reference \\
\hline RA $(1950)$ & 010443 & NED \\
Decl. $(1950)$ & +162501 & NED \\
Galactic latitude & -46.02 & UGC \\
$E_{B-V}$ & 0.10 & Burstein \& Heiles 1982 \\
distance & $0.27 D_{\text {Virgo }}$ & Kran-Korteweg 1986 \\
& $\sim 6 \mathrm{Mpc}$ & \\
distance $[\mathrm{Mpc}]$ & $5.5 \pm 30 \%$ & this paper \\
HI $S_{\nu} d \nu[\mathrm{Jy} \mathrm{km} / \mathrm{s}]$ & 12.6 & Lu et al. 1993 \\
HI velocity $[\mathrm{km} / \mathrm{s}]$ & $157 \pm 1$ & Lu et al. 1993 \\
HI W50 width $[\mathrm{km} / \mathrm{s}]$ & $73 \pm 1$ & Lu et al. 1993 \\
HI mass $\left[M_{\odot}\right]$ & $8.510^{7}$ & Hoffman et al. 1996 \\
$V_{\text {max }}[\mathrm{km} / \mathrm{s}]$ & 75 & Hoffman et al. 1996 \\
FIR Iras flux & below limits & Schmidt \& Boller 1992a \\
& & \\
$B$ & $14.55 \pm 0.08$ & this paper \\
$M_{B, 25.0}$ & -14.48 & this paper \\
$M_{B, \text { asym }}$ & -14.92 & this paper \\
$B-R$ & $+0.97 \pm 0.05$ & this paper \\
$B-I$ & +1.39 & Lu et al. 1993 and here \\
$B-J$ & $+1.55 \pm 0.15$ & this paper \\
$\mu_{0, B}\left[\mathrm{mag} / \square^{\prime \prime}\right]$ & $21.57 \pm 0.09$ & this paper \\
$\mu_{0, R}\left[\mathrm{mag} / \square^{\prime \prime}\right]$ & $20.65 \pm 0.06$ & this paper \\
$\mu_{0, J}\left[\mathrm{mag} / \square^{\prime \prime}\right]$ & $20.11 \pm 0.10$ & this paper \\
scale length $B[\mathrm{pc}]$ & $338 \pm 16$ & this paper \\
scale length $R[\mathrm{pc}]$ & $330 \pm 26$ & this paper \\
scale length $J[\mathrm{pc}]$ & $338 \pm 21$ & this paper \\
$a_{26.5 \mathrm{mag} / \square^{\prime \prime}}$ & $55^{\prime \prime}(1.46 \mathrm{kpc})$ & this paper \\
Elipticity & 0.33 & this paper \\
Number of HII regions & 5 & this paper \\
log $L_{\mathrm{H} \alpha}\left[\mathrm{erg} \mathrm{s}{ }^{-1}\right]$ & 38.6 & this paper \\
\hline & & \\
\hline
\end{tabular}

$(0.65 \mathrm{kpc})$ diameter. This blob of HII regions is off-set from the center by $11^{\prime \prime}(0.31 \mathrm{kpc})$ or about one scale length of the stellar light distribution. Very little diffuse $\mathrm{H} \alpha$ emission outside the HII regions is visible, but the image which was taken for selecting proper long slit settings might have an exposure time which was too short to detect very faint structures like those describes for example in the case of
NGC 4449 by Bomans et al. (1997). The long slit spectra along the major axis of UGC 685 cross some of the identified HII regions and indeed reveal the typical emission line pattern of HII regions. Outside these HII regions, but still well inside the low surface brightness envelope, sources with the shape of the point-spread function are visible. These objects trace the resolved brightest stars - mostly 


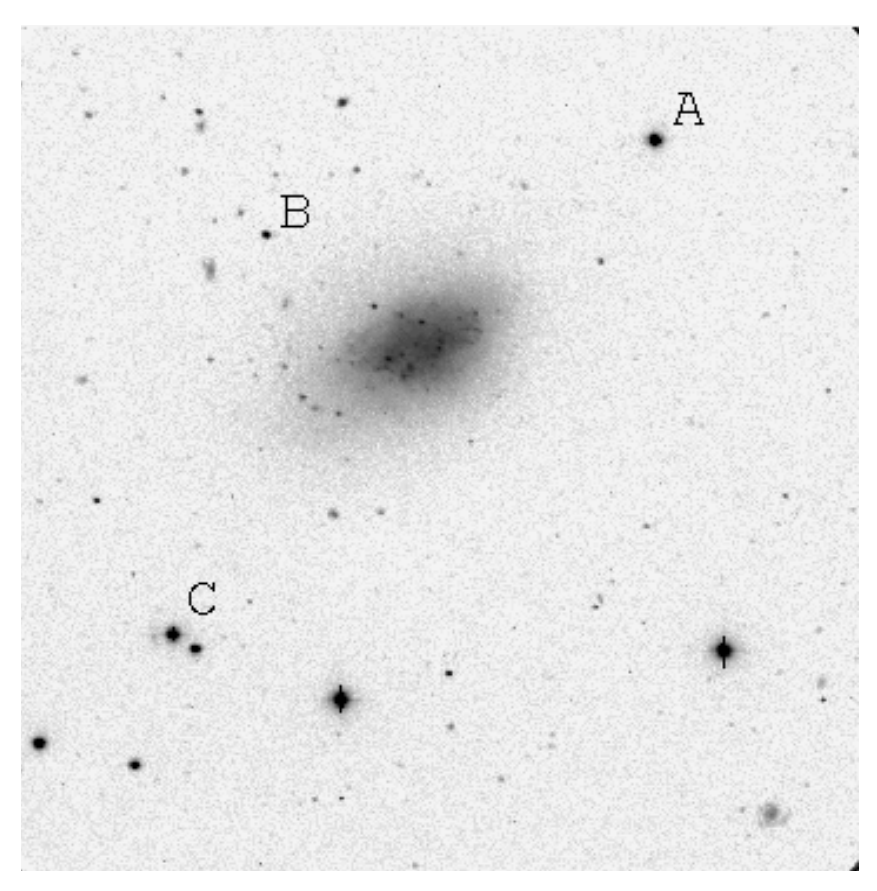

Fig. 1. CCD $3.5 \mathrm{~m}$ telescope $B$ image of UGC 685 . North top, East left. The horizontal side length is $346^{\prime \prime}$. The letters identify those very bright stars where residuals are still visible in Fig. 2

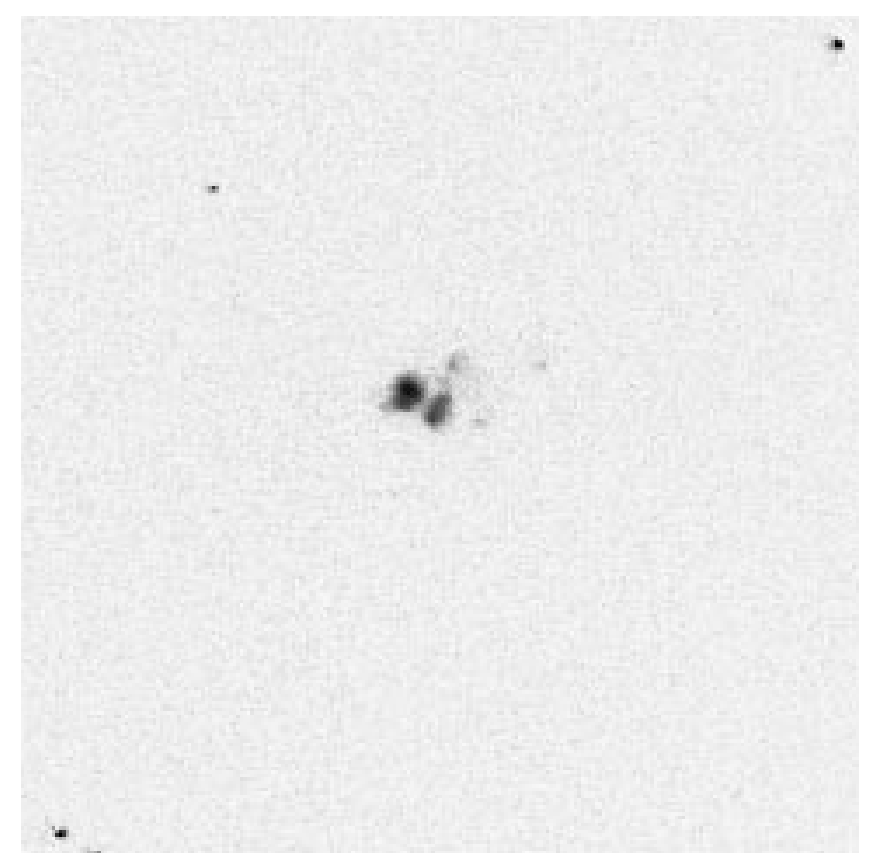

Fig. 2. CCD $2.2 \mathrm{~m}$ telescope image in $\mathrm{H} \alpha$ of UGC 685 after continuum subtraction. North top, East left. The side length is $160^{\prime \prime}$. The residuals seen at large distances from UGC 685 are from saturated stars which are identified in Fig. 1 by letters

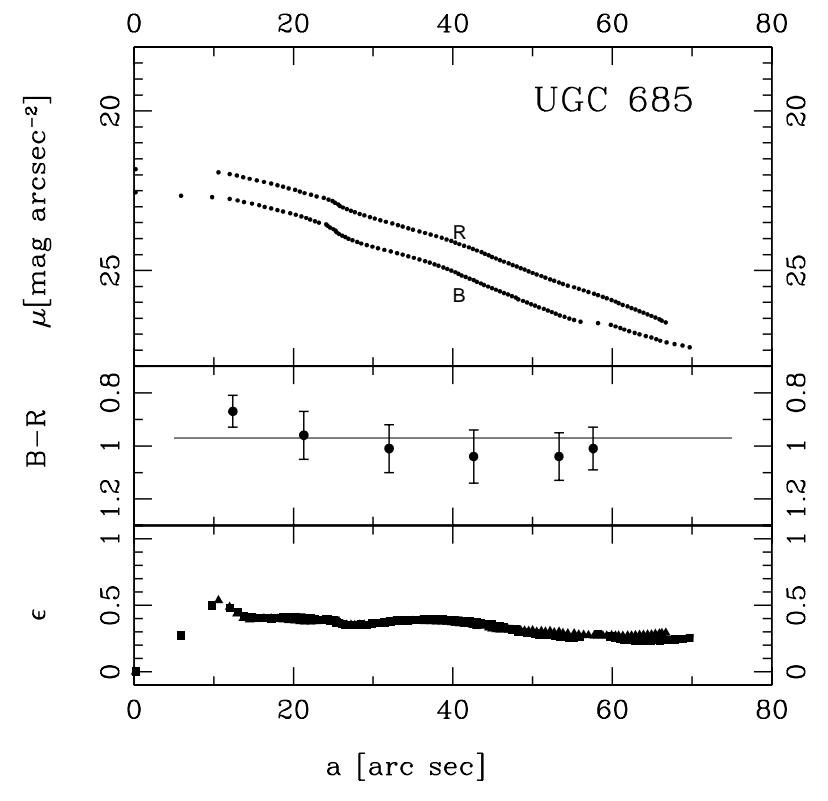

Fig. 3. Top: The surface brightness profiles according to the ellipticity fits in the red (upper) and blue (lower) $3.5 \mathrm{~m} \mathrm{CCD}$ frames. Middle: The color gradient as calculated from the curves in the upper graph. The straight line gives the color for the total light. Bottom: The radial ellipticity variation derived from the blue (squares) and red (triangles) frames (all $3.5 \mathrm{~m}$ telescope CCD data)

supergiants - in UGC 685 even though it is easily possible that some of these "stars" may be in reality composed objects like blends of stars or stellar clusters.

A mask was constructed at the $25.0 \mathrm{mag} / \square^{\prime \prime}$ level in $B$ and the flux in $B$ and $R$ inside this isophote was determined, yielding $B=14.55$ and $B-R=+0.97$ (Table 2). Errors are less than 0.1 and are mostly of systematic nature. The $B$ magnitude is in good agreement with those in the literature (see Schmidt \& Boller 1992a). The absolute values derived from these observed magnitudes were computed using the distance estimate of $5.5 \mathrm{Mpc}$ from chapter 3.4 and a correction for galactic foreground extinction as given by Burstein \& Heiles (1982). No attempt was done to correct for internal reddening. As already mentioned, the $J$ frames exclude a small part of the faint eastern outskirt, less than $4 \%$ of the total area. These outskirts contribute little light, nevertheless the derived $J$ magnitude of 13.00 is more like a good lower limit to the total flux.

I measured the flux in the continuum-free $\mathrm{H} \alpha$ image and found a flux of $110^{-13} \mathrm{erg} \mathrm{s}^{-1} \mathrm{~cm}^{-2}$ which converts to an absolute flux in the $\mathrm{H} \alpha$ line as given in Table 2 (using $D=5.5 \mathrm{Mpc}$ ). The Poisson error of this value is small, less than $1 \%$, but almost negligible compared to the error of the zero point calibration of about $20 \%$. Hunter et al. (1994) observed $\mathrm{H} \alpha$ line fluxes for a sample of latetype galaxies, mostly dwarf irregulars. In comparison to this sample, the $\mathrm{H} \alpha$ line flux of UGC 685 is normal for 
an irregular dwarf of its total magnitude and indicates a relatively low recent star formation rate (see below).

\subsection{Surface photometry}

Figure 3 shows the surface brightness profile, the radial color profil and the variation of the ellipticity with major axis for $B$ and $R$. UGC 685 has a rather elliptical shape with a mean ellipticity value of 0.33 for both colors. The ellipticity varies only slightly with radius while the (not shown) position angle increases steadily by the small, but significant amount of 20 degree from 10 to $70^{\prime \prime}$. The surface brightness profile can be traced out to about $70^{\prime \prime}$ in $B$ and $65^{\prime \prime}$ in $R$. It is interesting to note that in neutral hydrogen, the profile of UGC 685 was traced to a 2.6 times larger radius than here in the optical (see Hoffman et al. 1996 for the HI data). In $J$, the galaxy was traced down to $23.1 \mathrm{mag} / \square^{\prime \prime}$ and to a distance of $35^{\prime \prime}$. Within this more limited range, the ellipticity is essentially the same $(0.32)$ as in the CCD color bands.

In all three colors, the profile can be well described by an exponential law outside a central region of about $10^{\prime \prime}$. The central profiles are significantly flatter. One should remember that in the very center, several knots (HII regions, stars, clusters) were removed, therefore it is difficult to trace the profile there. Outside $10^{\prime \prime}$, I fitted an exponential brightness profile to the data and got a scale length of $12.4^{\prime \prime}$ to $12.7^{\prime \prime}$ (Table 2). Thus, the scale length of the stellar distribution is 10 times smaller than the one of the HI distribution (Hoffman et al. 1996). The obtained parameters, already converted to metric values with the distance estimated below, are given in Table 2 . They are quite normal for a galaxy of this size and luminosity ${ }^{1}$. The central surface brightnesses are relatively high for an irregular dwarf with only moderate signs of recent star formation. A color profile was calculated (Fig. 3). Most of the body has the same color while the central part is slightly bluer. $B-J$, albeit having a larger systematic error, shows no significant variation with radius.

The exponential law parameters were used to calculate asymptotic total magnitudes of $14.11,13.14$, and 12.60 in $B, R$, and $J$, respectively.

$\mathrm{H} \alpha$ emission was only detected in a small area near to the center of UGC 685 , slightly off towards the southeast. Thus, the recent places of star formation activity show a strong asymmetry in the angular distribution and are well localized. I measured the $\mathrm{H} \alpha$ surface brightness as a function of radius and averaged over the azimuth (for a better comparison with similar profiles for other irregular galaxies presented by Hunter et al. 1998). Naturally, this radial $\mathrm{H} \alpha$ profile is only valid for the small sector

\footnotetext{
${ }^{1}$ The $R$ band data from the $2.2 \mathrm{~m}$ telescope yielded very similar results: The profile is detected out to about $55^{\prime \prime}$, is flat in the center, and shows an exponential distribution law with a scale length of $16 \pm 1.2^{\prime \prime}$.
}

(Fig. 2). Outside, the $\mathrm{H} \alpha$ surface brightness is below the detection limits (about $1.51^{-16} \mathrm{erg} \mathrm{s}^{-1} \mathrm{~cm}^{-2}$ ). Assume that the $\mathrm{H} \alpha$ flux is a good measure of the total number of ionizing photons emitted in a galaxy. This number can be compared to the photons expected from massive stars for a given initial mass function (IMF). Taking into account the lifetimes of the massive stars, a formation rate of hot stars can be derived and extended to a total star formation rate (SFR) by extrapolating to all stars with the IMF. Hunter \& Gallagher (1986, see also Gallagher et al. 1984) used a Salpeter function to establish a conversion formula which I used to transform the observed $\mathrm{H} \alpha$ surface brightness of UGC 685 into a star formation rate per $\mathrm{pc}^{2}$. A distance of $5.5 \mathrm{Mpc}$ was used. Figure 4 show the radial profile. Given the uncertainty in the $\mathrm{H} \alpha$ flux calibration, the only secure feature is the off-center peak in the recent specific star formation activity, and an overall trend that the recent star formation activity drops to larger radii. The shape of the radial distribution of the recent star formation activity in UGC 685 is similar to the one observed in Sex A and IC 1613 (Hunter et al. 1998), even though its overall value is lower than in those two dwarfs. As discussed in more detail by van Zee et al. (1998), an azimutal average of the local SFR (as measured by the $\mathrm{H} \alpha$ flux) can be misleading for irregular dwarf galaxies where the star formation often takes place at only a few places. UGC 685 is obviously a good example for this case. Using again the derivation of Hunter \& Gallagher (1986), the total $\mathrm{H} \alpha$ flux from chapter 3.1 can be transformed into an average SFR over the last $\sim 10^{7}$ yr. I derived $0.003 M_{\odot} \mathrm{yr}^{-1}$ with an error based on the measurement alone of about $20 \%$. Here again, the result depends on the applied distance value of $5.5 \mathrm{Mpc}$.

The radial color gradient shows no indication that the average SFR varies strongly with location (Fig. 3). A $B-R$ color map shows a quite homogeneous distribution with only small ( $5 \%$ level) deviations in the central area, where recent (and localized) star formation is already indicated by the HII regions.

\subsection{Resolved stellar population}

A total of 209 stars are detected in both $(B$ and $R$ ) frames, down to a limiting magnitude of 24 in both filters. These stars are identified in Table $3^{2}$. Fainter than 22.5 in $R$ and 23.0 in $B$, the photometric errors as estimated by the DAOPHOT PSF fitting algorithm are larger than 0.1. 73 of these stars are projected on or belong to UGC 685. As the CCD frames are by far larger than the galaxy, I can determine the surface density of fore- and background objects with stellar PSF. A statistical correction of 5.1 stars was calculated which leaves about 68 stars belonging to

\footnotetext{
2 An upper flux limit had been set when running DAOPHOT to avoid saturated stars. Therefore, the brightest stars visible in Fig. 1 cannot be found in Table 3, but they are Milky Way objects anyway.
} 


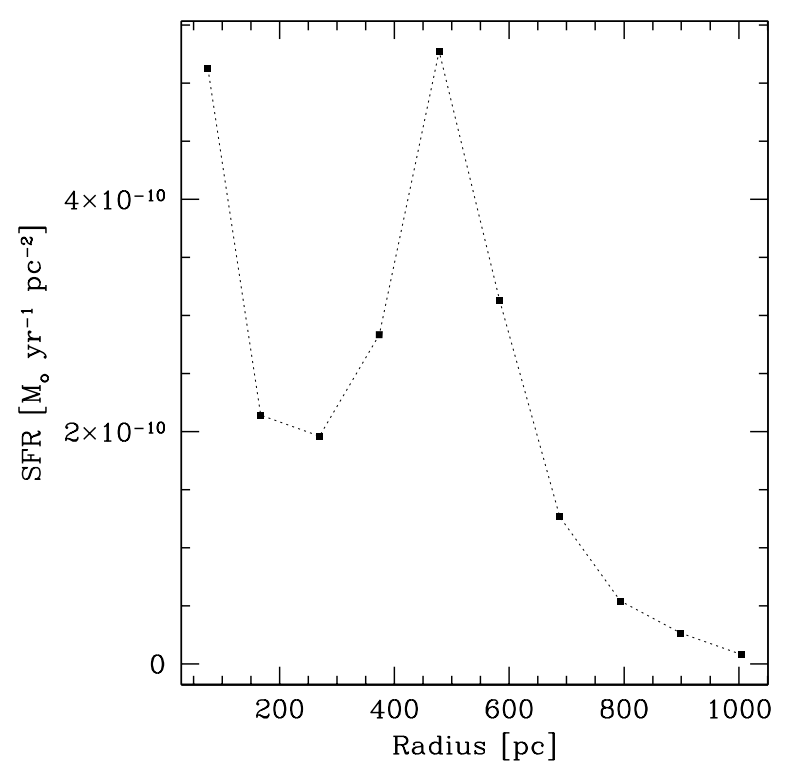

Fig. 4. The radial distribution of the recent star formation activity as derived from the $\mathrm{H} \alpha$ flux applying the transformation relation between flux and star formation rate as given by Hunter \& Gallagher (1986). A distance of $5.5 \mathrm{Mpc}$ was assumed. This curve is only valid for the sector where HII regions are visible, outside this sector the star formation rate is below the detection limit

UGC 685. The color distribution of the field stars outside UGC 685 (see Fig. 5) shows that most of the contamination contributes in the red while practically all detected blue objects $(B-R<0.5)$ belong to the young population in UGC 685.

Artificial star tests were applied inside DAOPHOT to estimate the completeness of the star detection especially in the magnitude range of the supergiants of UGC 685 . 50 stars were added in 1 magnitude bins in several experiments for each of these bins. The recovery rate indicates that the completeness starts to drop from almost $100 \%$ at 21.9 (22.7) in $R(\mathrm{~B})$ and the $50 \%$ completeness magnitudes are about $22.9(23.7)$ in $R(\mathrm{~B})$.

Figure 5 shows the color-magnitude diagram of the UGC 685. The left part shows all 209 detected points sources and indicates the $50 \%$ completeness limit while the right panel presents only those 173 stars where the photometric errors are less than 0.2 in $B$ and $R$. The open symbols indicate the 136 sources which belong to the field around UGC 685 while the filled dots indicate the 73 sources in UGC 685 . 56 of the UGC 685 objects have photometric errors less than 0.2 in both bands.

A comparison with Geneva evolutionary tracks (Fig. 6) as well as with the Padua isochrones (Meynet et al. 1994; Bertelli et al. 1994) indicates that several blue supergiants and fewer yellow supergiants have been resolved in the mass range 15 to $60 M_{\odot}$ (ZAMS). Given the higher field
Table 3. Stars found in $B$ and $R$ on the UGC 685 frames. Given are a running number (from $B$ frame), relative frame coordinates in arc sec where $+x$ increases towards East and $+y$ increases towards South, the $B$ magnitudes and their errors according to DAOPHOT (which does not include systematic errors of the calibrations) followed by the same photometric informations for $R$, and finally $B-R$

\begin{tabular}{|c|c|c|c|c|c|c|c|}
\hline No. & $\begin{array}{r}X \\
\operatorname{arcsec}\end{array}$ & $\begin{array}{r}Y \\
\operatorname{arcsec}\end{array}$ & $\begin{array}{r}B \\
\text { mag }\end{array}$ & $\begin{array}{r}\sigma_{B} \\
\mathrm{mag}\end{array}$ & $\begin{array}{r}R \\
\text { mag }\end{array}$ & $\begin{array}{r}\sigma_{R} \\
\mathrm{mag}\end{array}$ & $\begin{array}{r}B-R \\
\text { mag }\end{array}$ \\
\hline 12 & 323.7 & 11.2 & 22.19 & 0.05 & 21.08 & 0.06 & 1.11 \\
\hline 13 & 280.5 & 11.6 & 22.85 & 0.05 & 20.63 & 0.08 & 2.22 \\
\hline 18 & 106.1 & 16.4 & 23.77 & 0.21 & 22.42 & 0.11 & 1.35 \\
\hline 29 & 221.1 & 28.6 & 23.58 & 0.11 & 21.39 & 0.07 & 2.19 \\
\hline 31 & 287.6 & 29.6 & 21.94 & 0.05 & 20.22 & 0.04 & 1.73 \\
\hline 32 & 213.5 & 31.6 & 20.14 & 0.05 & 18.74 & 0.05 & 1.40 \\
\hline 35 & 3.4 & 32.9 & 23.38 & 0.13 & 21.54 & 0.09 & 1.84 \\
\hline 39 & 272.6 & 35.7 & 20.28 & 0.02 & 18.91 & 0.02 & 1.37 \\
\hline 40 & 318.5 & 36.9 & 21.67 & 0.04 & 20.08 & 0.05 & 1.59 \\
\hline 45 & 271.9 & 41.5 & 21.72 & 0.07 & 20.78 & 0.09 & 0.93 \\
\hline 52 & 2.0 & 53.1 & 22.80 & 0.09 & 20.87 & 0.08 & 1.93 \\
\hline 53 & 108.7 & 56.7 & 23.52 & 0.11 & 21.99 & 0.07 & 1.53 \\
\hline 55 & 181.2 & 60.1 & 23.42 & 0.16 & 21.50 & 0.07 & 1.91 \\
\hline 56 & 207.6 & 59.7 & 21.30 & 0.03 & 20.39 & 0.03 & 0.91 \\
\hline 58 & 278.5 & 60.1 & 21.83 & 0.04 & 20.81 & 0.03 & 1.02 \\
\hline 59 & 218.1 & 61.5 & 22.72 & 0.07 & 21.10 & 0.07 & 1.62 \\
\hline 60 & 318.8 & 61.0 & 23.80 & 0.23 & 21.67 & 0.09 & 2.13 \\
\hline 62 & 241.1 & 61.8 & 22.34 & 0.07 & 21.33 & 0.07 & 1.01 \\
\hline 66 & 178.0 & 65.5 & 22.22 & 0.05 & 21.86 & 0.07 & 0.37 \\
\hline 68 & 304.9 & 67.1 & 23.35 & 0.14 & 21.78 & 0.09 & 1.56 \\
\hline 67 & 138.3 & 66.9 & 22.55 & 0.06 & 21.15 & 0.09 & 1.40 \\
\hline 69 & 6.2 & 67.9 & 22.26 & 0.05 & 20.60 & 0.06 & 1.66 \\
\hline 79 & 158.0 & 75.4 & 23.31 & 0.14 & 21.86 & 0.12 & 1.45 \\
\hline 81 & 160.3 & 77.4 & 23.32 & 0.13 & 22.79 & 0.18 & 0.53 \\
\hline 82 & 57.8 & 78.1 & 23.06 & 0.07 & 21.59 & 0.09 & 1.47 \\
\hline 83 & 255.6 & 77.6 & 21.66 & 0.07 & 20.88 & 0.09 & 0.77 \\
\hline 87 & 266.5 & 80.8 & 22.45 & 0.04 & 20.72 & 0.03 & 1.73 \\
\hline 91 & 244.8 & 86.7 & 18.73 & 0.02 & 17.16 & 0.03 & 1.58 \\
\hline 95 & 199.8 & 91.2 & 23.25 & 0.11 & 21.66 & 0.08 & 1.59 \\
\hline 96 & 26.5 & 94.2 & 22.69 & 0.07 & 22.00 & 0.10 & 0.70 \\
\hline 97 & 165.2 & 94.5 & 22.67 & 0.10 & 20.87 & 0.06 & 1.81 \\
\hline 98 & 106.7 & 97.8 & 20.94 & 0.02 & 20.06 & 0.03 & 0.88 \\
\hline 99 & 270.5 & 98.6 & 22.52 & 0.09 & 21.27 & 0.09 & 1.26 \\
\hline 104 & 267.9 & 101.4 & 21.62 & 0.09 & 19.74 & 0.08 & 1.87 \\
\hline 111 & 222.3 & 107.0 & 22.81 & 0.11 & 20.59 & 0.03 & 2.22 \\
\hline 113 & 246.3 & 108.1 & 24.09 & 0.19 & 22.98 & 0.18 & 1.11 \\
\hline 114 & 324.0 & 107.9 & 23.42 & 0.11 & 22.14 & 0.10 & 1.28 \\
\hline 123 & 236.5 & 114.3 & 22.45 & 0.08 & 20.25 & 0.06 & 2.20 \\
\hline 124 & 157.4 & 114.8 & 23.50 & 0.15 & 22.27 & 0.11 & 1.22 \\
\hline 128 & 31.1 & 116.5 & 22.68 & 0.07 & 20.82 & 0.05 & 1.86 \\
\hline 133 & 200.5 & 116.4 & 20.22 & 0.02 & 19.29 & 0.02 & 0.94 \\
\hline 134 & 237.3 & 116.1 & 22.21 & 0.05 & 20.06 & 0.06 & 2.15 \\
\hline 135 & 144.2 & 117.6 & 24.13 & 0.28 & 23.27 & 0.24 & 0.86 \\
\hline 138 & 149.4 & 118.4 & 22.68 & 0.06 & 21.48 & 0.07 & 1.20 \\
\hline 143 & 158.7 & 119.4 & 21.66 & 0.04 & 20.97 & 0.03 & 0.70 \\
\hline 145 & 39.3 & 120.2 & 22.80 & 0.09 & 21.87 & 0.10 & 0.93 \\
\hline 146 & 189.2 & 120.3 & 21.11 & 0.03 & 20.65 & 0.05 & 0.46 \\
\hline 149 & 165.2 & 121.4 & 21.77 & 0.04 & 21.43 & 0.05 & 0.34 \\
\hline 157 & 184.6 & 123.9 & 22.71 & 0.09 & 22.57 & 0.20 & 0.14 \\
\hline 155 & 180.9 & 123.1 & 20.54 & 0.02 & 19.61 & 0.01 & 0.92 \\
\hline 165 & 158.8 & 125.9 & 22.05 & 0.04 & 21.39 & 0.06 & 0.66 \\
\hline 166 & 161.9 & 125.5 & 22.00 & 0.08 & 21.62 & 0.11 & 0.37 \\
\hline 169 & 173.7 & 126.2 & 21.91 & 0.04 & 21.62 & 0.08 & 0.30 \\
\hline 171 & 156.7 & 126.7 & 22.15 & 0.06 & 22.26 & 0.12 & -0.11 \\
\hline 176 & 171.9 & 128.7 & 22.18 & 0.08 & 20.82 & 0.06 & 1.36 \\
\hline
\end{tabular}




\begin{tabular}{|c|c|c|c|c|c|c|c|}
\hline No. & $\begin{array}{r}X \\
\operatorname{arcsec}\end{array}$ & $\begin{array}{r}Y \\
\operatorname{arcsec}\end{array}$ & $\begin{array}{r}B \\
\text { mag }\end{array}$ & $\begin{array}{r}\sigma_{B} \\
\mathrm{mag}\end{array}$ & $\begin{array}{r}R \\
\mathrm{mag}\end{array}$ & $\begin{array}{r}\sigma_{R} \\
\mathrm{mag}\end{array}$ & $\begin{array}{r}B-R \\
\mathrm{mag}\end{array}$ \\
\hline 180 & 68.5 & 129.3 & 23.25 & 0.13 & 21.81 & 0.06 & 1.44 \\
\hline 186 & 167.9 & 130.2 & 22.43 & 0.06 & 21.69 & 0.10 & 0.74 \\
\hline 189 & 173.9 & 130.7 & 21.77 & 0.04 & 21.24 & 0.07 & 0.52 \\
\hline 193 & 162.6 & 130.9 & 22.46 & 0.07 & 21.61 & 0.11 & 0.85 \\
\hline 194 & 172.4 & 131.3 & 21.42 & 0.05 & 21.27 & 0.04 & 0.16 \\
\hline 195 & 189.2 & 131.0 & 23.31 & 0.10 & 22.35 & 0.14 & 0.97 \\
\hline 198 & 209.9 & 132.6 & 22.29 & 0.06 & 21.88 & 0.07 & 0.41 \\
\hline 200 & 179.2 & 133.2 & 22.34 & 0.06 & 21.55 & 0.06 & 0.79 \\
\hline 203 & 165.0 & 133.5 & 24.14 & 0.29 & 22.11 & 0.16 & 2.02 \\
\hline 206 & 222.8 & 133.6 & 22.03 & 0.04 & 21.36 & 0.04 & 0.67 \\
\hline 207 & 173.8 & 134.1 & 20.67 & 0.03 & 20.15 & 0.03 & 0.52 \\
\hline 208 & 180.1 & 134.3 & 22.51 & 0.07 & 21.12 & 0.05 & 1.39 \\
\hline 212 & 195.6 & 135.5 & 23.11 & 0.14 & 22.59 & 0.17 & 0.51 \\
\hline 214 & 209.4 & 134.7 & 22.66 & 0.11 & 22.00 & 0.10 & 0.66 \\
\hline 216 & 188.9 & 136.3 & 21.23 & 0.02 & 19.55 & 0.02 & 1.68 \\
\hline 217 & 175.7 & 136.8 & 21.73 & 0.04 & 21.32 & 0.06 & 0.40 \\
\hline 221 & 182.1 & 137.7 & 23.54 & 0.22 & 22.14 & 0.15 & 1.40 \\
\hline 222 & 204.4 & 138.2 & 22.48 & 0.08 & 21.29 & 0.05 & 1.19 \\
\hline 223 & 92.9 & 138.4 & 23.38 & 0.13 & 21.65 & 0.05 & 1.73 \\
\hline 226 & 251.9 & 138.7 & 23.37 & 0.12 & 21.64 & 0.05 & 1.73 \\
\hline 227 & 268.1 & 138.7 & 21.90 & 0.05 & 21.03 & 0.07 & 0.87 \\
\hline 229 & 215.3 & 138.9 & 22.04 & 0.07 & 21.98 & 0.06 & 0.06 \\
\hline 232 & 209.5 & 140.2 & 21.85 & 0.04 & 21.49 & 0.04 & 0.35 \\
\hline 233 & 176.2 & 140.8 & 22.48 & 0.06 & 22.71 & 0.20 & -0.22 \\
\hline 238 & 198.6 & 142.4 & 21.79 & 0.05 & 21.36 & 0.05 & 0.43 \\
\hline 237 & 237.3 & 141.6 & 21.67 & 0.03 & 20.82 & 0.04 & 0.85 \\
\hline 239 & 205.4 & 142.0 & 22.91 & 0.14 & 22.30 & 0.15 & 0.61 \\
\hline 241 & 200.7 & 142.6 & 22.67 & 0.09 & 22.32 & 0.10 & 0.34 \\
\hline 243 & 173.0 & 143.4 & 23.21 & 0.15 & 22.94 & 0.24 & 0.27 \\
\hline 247 & 177.5 & 143.7 & 23.71 & 0.18 & 22.92 & 0.29 & 0.80 \\
\hline 245 & 193.0 & 143.4 & 23.27 & 0.29 & 22.32 & 0.13 & 0.95 \\
\hline 246 & 168.9 & 143.8 & 22.92 & 0.13 & 21.83 & 0.10 & 1.09 \\
\hline 249 & 249.6 & 145.1 & 22.45 & 0.06 & 21.81 & 0.08 & 0.64 \\
\hline 250 & 188.3 & 145.9 & 21.10 & 0.05 & 21.06 & 0.07 & 0.04 \\
\hline 262 & 320.8 & 147.2 & 22.05 & 0.07 & 20.59 & 0.07 & 1.46 \\
\hline 263 & 180.0 & 148.7 & 23.52 & 0.14 & 22.39 & 0.16 & 1.13 \\
\hline 265 & 203.5 & 150.1 & 22.75 & 0.09 & 22.23 & 0.08 & 0.52 \\
\hline 269 & 178.6 & 151.0 & 22.08 & 0.05 & 21.77 & 0.09 & 0.31 \\
\hline 270 & 12.9 & 151.3 & 21.77 & 0.04 & 19.92 & 0.03 & 1.85 \\
\hline 271 & 207.0 & 151.4 & 23.99 & 0.21 & 22.20 & 0.08 & 1.79 \\
\hline 273 & 149.0 & 153.7 & 23.10 & 0.10 & 22.23 & 0.10 & 0.88 \\
\hline 274 & 230.0 & 154.2 & 20.90 & 0.03 & 19.96 & 0.03 & 0.95 \\
\hline 279 & 224.9 & 158.8 & 22.08 & 0.06 & 20.68 & 0.10 & 1.40 \\
\hline 282 & 215.1 & 161.3 & 20.97 & 0.02 & 20.02 & 0.02 & 0.95 \\
\hline 286 & 69.3 & 164.8 & 23.24 & 0.12 & 21.80 & 0.10 & 1.44 \\
\hline 290 & 65.5 & 166.3 & 23.35 & 0.12 & 21.87 & 0.08 & 1.49 \\
\hline 293 & 245.5 & 167.6 & 23.88 & 0.21 & 22.79 & 0.18 & 1.09 \\
\hline 297 & 160.4 & 172.7 & 21.86 & 0.04 & 20.82 & 0.04 & 1.03 \\
\hline 302 & 247.2 & 175.9 & 22.45 & 0.06 & 20.63 & 0.03 & 1.82 \\
\hline 304 & 276.8 & 179.4 & 23.61 & 0.14 & 22.57 & 0.14 & 1.03 \\
\hline 306 & 211.7 & 180.1 & 22.79 & 0.07 & 21.51 & 0.05 & 1.28 \\
\hline 310 & 246.9 & 185.4 & 24.06 & 0.22 & 22.72 & 0.13 & 1.34 \\
\hline 313 & 330.5 & 189.1 & 22.97 & 0.09 & 22.03 & 0.10 & 0.94 \\
\hline 317 & 30.8 & 195.2 & 21.58 & 0.03 & 19.87 & 0.04 & 1.72 \\
\hline 320 & 315.0 & 197.2 & 19.56 & 0.03 & 17.75 & 0.06 & 1.81 \\
\hline 323 & 338.5 & 197.5 & 22.46 & 0.06 & 20.74 & 0.04 & 1.71 \\
\hline 325 & 2.6 & 201.0 & 23.17 & 0.08 & 22.25 & 0.14 & 0.91 \\
\hline 327 & 197.8 & 202.1 & 21.80 & 0.03 & 21.05 & 0.03 & 0.76 \\
\hline 328 & 26.0 & 202.7 & 23.35 & 0.08 & 21.89 & 0.07 & 1.46 \\
\hline 331 & 216.9 & 203.0 & 21.25 & 0.06 & 20.36 & 0.08 & 0.89 \\
\hline 336 & 33.7 & 207.1 & 23.44 & 0.12 & 22.11 & 0.08 & 1.33 \\
\hline 337 & 88.0 & 207.9 & 21.55 & 0.05 & 19.70 & 0.04 & 1.84 \\
\hline
\end{tabular}

\begin{tabular}{|c|c|c|c|c|c|c|c|}
\hline No. & $\begin{array}{r}X \\
\operatorname{arcsec}\end{array}$ & $\begin{array}{r}Y \\
\operatorname{arcsec}\end{array}$ & $\begin{array}{r}B \\
\text { mag }\end{array}$ & $\begin{array}{r}\sigma_{B} \\
\text { mag }\end{array}$ & $\begin{array}{r}R \\
\text { mag }\end{array}$ & $\begin{array}{r}\sigma_{R} \\
\mathrm{mag}\end{array}$ & $\begin{array}{r}B-R \\
\text { mag }\end{array}$ \\
\hline 340 & 300.9 & 210.1 & 23.89 & 0.20 & 21.80 & 0.08 & 2.09 \\
\hline 342 & 125.2 & 212.0 & 23.11 & 0.08 & 21.87 & 0.09 & 1.24 \\
\hline 343 & 44.4 & 213.9 & 23.39 & 0.09 & 21.93 & 0.11 & 1.46 \\
\hline 345 & 273.3 & 216.0 & 23.05 & 0.06 & 21.47 & 0.08 & 1.58 \\
\hline 349 & 178.3 & 220.8 & 23.41 & 0.07 & 21.95 & 0.09 & 1.46 \\
\hline 351 & 134.5 & 224.3 & 24.10 & 0.23 & 22.85 & 0.19 & 1.25 \\
\hline 353 & 300.2 & 227.9 & 21.99 & 0.05 & 20.98 & 0.08 & 1.01 \\
\hline 354 & 56.7 & 229.2 & 23.39 & 0.09 & 22.01 & 0.09 & 1.38 \\
\hline 356 & 102.1 & 231.0 & 22.67 & 0.06 & 21.96 & 0.08 & 0.70 \\
\hline 357 & 6.9 & 232.2 & 22.76 & 0.09 & 21.65 & 0.07 & 1.10 \\
\hline 358 & 24.5 & 232.2 & 23.80 & 0.21 & 21.63 & 0.07 & 2.18 \\
\hline 361 & 199.6 & 234.5 & 23.72 & 0.17 & 22.50 & 0.12 & 1.22 \\
\hline 366 & 107.5 & 237.5 & 22.19 & 0.08 & 21.35 & 0.06 & 0.84 \\
\hline 372 & 251.9 & 239.4 & 22.32 & 0.07 & 21.42 & 0.07 & 0.91 \\
\hline 375 & 109.1 & 241.6 & 20.93 & 0.05 & 19.31 & 0.05 & 1.62 \\
\hline 380 & 233.7 & 247.2 & 23.03 & 0.09 & 21.59 & 0.07 & 1.44 \\
\hline 384 & 249.6 & 247.9 & 24.72 & 0.30 & 23.40 & 0.26 & 1.32 \\
\hline 387 & 303.2 & 248.1 & 23.48 & 0.13 & 22.13 & 0.12 & 1.35 \\
\hline 385 & 89.2 & 248.2 & 22.22 & 0.07 & 21.27 & 0.07 & 0.96 \\
\hline 391 & 16.9 & 251.3 & 23.06 & 0.11 & 21.79 & 0.10 & 1.27 \\
\hline 396 & 262.6 & 254.3 & 23.64 & 0.16 & 22.20 & 0.07 & 1.44 \\
\hline 400 & 166.7 & 261.7 & 23.57 & 0.14 & 21.79 & 0.06 & 1.79 \\
\hline 402 & 1.1 & 262.8 & 23.24 & 0.12 & 22.64 & 0.14 & 0.61 \\
\hline 405 & 227.4 & 264.3 & 23.45 & 0.15 & 22.37 & 0.13 & 1.09 \\
\hline 407 & 169.6 & 269.2 & 19.40 & 0.04 & 17.72 & 0.05 & 1.68 \\
\hline 409 & 128.4 & 271.3 & 23.47 & 0.13 & 21.27 & 0.06 & 2.21 \\
\hline 408 & 62.0 & 271.6 & 23.25 & 0.09 & 22.38 & 0.14 & 0.87 \\
\hline 410 & 15.9 & 272.9 & 22.40 & 0.08 & 20.66 & 0.07 & 1.74 \\
\hline 417 & 15.1 & 280.2 & 20.44 & 0.05 & 18.80 & 0.07 & 1.65 \\
\hline 418 & 277.1 & 282.8 & 23.59 & 0.16 & 22.20 & 0.09 & 1.39 \\
\hline 421 & 82.3 & 285.4 & 23.57 & 0.16 & 22.02 & 0.08 & 1.56 \\
\hline 424 & 314.4 & 287.2 & 23.74 & 0.17 & 22.54 & 0.15 & 1.20 \\
\hline 427 & 52.8 & 290.5 & 22.80 & 0.08 & 21.65 & 0.11 & 1.15 \\
\hline 428 & 168.9 & 291.4 & 21.66 & 0.05 & 20.60 & 0.07 & 1.07 \\
\hline 430 & 43.8 & 292.8 & 23.52 & 0.12 & 22.66 & 0.16 & 0.85 \\
\hline 432 & 126.9 & 294.7 & 23.26 & 0.10 & 21.77 & 0.08 & 1.49 \\
\hline 433 & 215.1 & 294.8 & 22.93 & 0.05 & 22.48 & 0.14 & 0.45 \\
\hline 436 & 36.8 & 297.6 & 23.58 & 0.14 & 21.53 & 0.09 & 2.04 \\
\hline 440 & 127.7 & 299.4 & 23.02 & 0.09 & 22.10 & 0.08 & 0.92 \\
\hline 444 & 322.7 & 303.2 & 23.92 & 0.25 & 22.53 & 0.14 & 1.39 \\
\hline 446 & 299.2 & 307.4 & 16.62 & 0.05 & 15.45 & 0.04 & 1.17 \\
\hline 445 & 190.3 & 307.2 & 23.20 & 0.09 & 22.22 & 0.13 & 0.98 \\
\hline 447 & 346.9 & 309.9 & 23.24 & 0.15 & 21.68 & 0.09 & 1.55 \\
\hline 451 & 148.0 & 313.2 & 22.37 & 0.07 & 20.87 & 0.07 & 1.50 \\
\hline 457 & 211.1 & 316.6 & 23.08 & 0.11 & 21.15 & 0.04 & 1.92 \\
\hline 461 & 73.4 & 319.8 & 23.33 & 0.12 & 22.26 & 0.10 & 1.07 \\
\hline 464 & 225.1 & 321.8 & 22.37 & 0.07 & 20.68 & 0.06 & 1.69 \\
\hline 465 & 231.2 & 323.7 & 23.32 & 0.10 & 22.20 & 0.13 & 1.12 \\
\hline 467 & 36.9 & 324.4 & 22.31 & 0.12 & 21.58 & 0.10 & 0.73 \\
\hline 471 & 48.5 & 325.9 & 22.43 & 0.07 & 21.35 & 0.07 & 1.08 \\
\hline 478 & 144.2 & 331.5 & 23.59 & 0.14 & 22.12 & 0.10 & 1.48 \\
\hline 485 & 45.8 & 336.9 & 23.22 & 0.13 & 22.28 & 0.11 & 0.94 \\
\hline 488 & 142.9 & 340.0 & 24.18 & 0.17 & 23.05 & 0.21 & 1.12 \\
\hline 499 & 183.8 & 349.1 & 22.58 & 0.07 & 21.77 & 0.07 & 0.81 \\
\hline
\end{tabular}




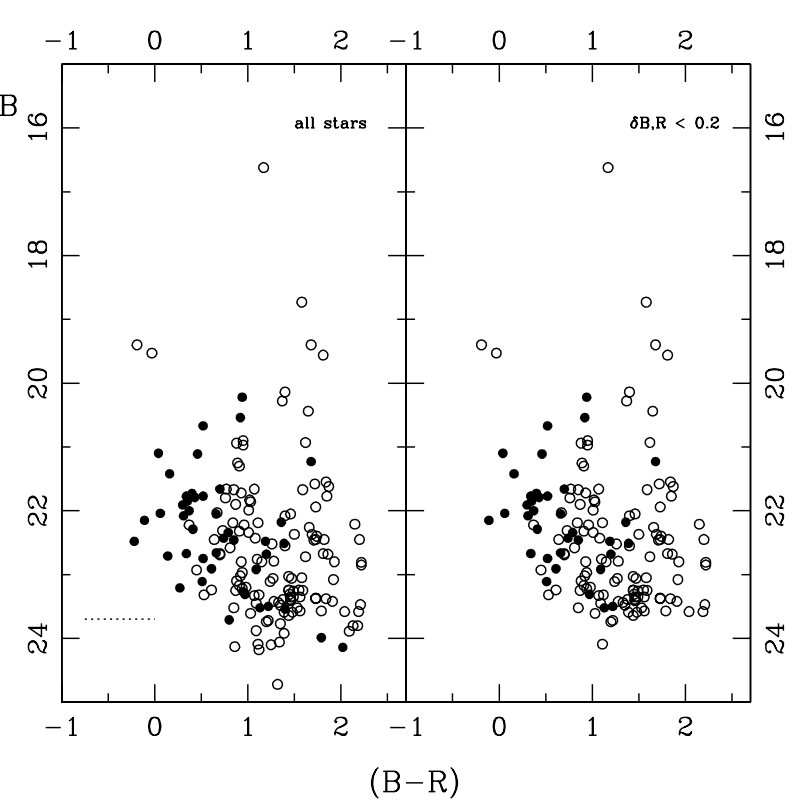

Fig. 5. Left: $B-R, B$ color-magnitude diagram of the 209 stars in the field of UGC 685 . The 136 stars outside the galaxy are marked as circles, those 73 of UGC 685 (or projected on UGC 685) are marked by filled dots. The dotted line indicates the $50 \%$ completeness limit (see text). Right: The panel shows only those 173 sources which have photometric errors in $B$ and $R$ less than 0.2 . 56 of these sources belong to UGC 685 . UGC 685 clearly shows a population of blue and yellow supergiants pointing to recent star formation activity. Some red supergiants seem be present too, but are harder to disentangle from the field confusion

contamination, is difficult to detect a red supergiant population. Again, one should remember that some of these objects, especially of the blue ones, might be unresolved clusters. Having only one color in most cases, clusters (or blends) can not be distinguished. Naturally, clusters of blue stars can be brighter than individual stars. Contrary, HII regions have been identified unambiguously with the $\mathrm{H} \alpha$ image.

\subsection{The distance of $U G C 685$}

The brightest blue supergiants (BBSG's) have a long tradition as distance indicators (K\&T, R\&RR). It is usual to determine the mean of the three brightest blue supergiants, $B_{3}$, and apply a calibration as provided by K\&T and $\mathrm{R} \& \mathrm{RR}$ to derive the distance modulus of the considered galaxy. The detailed analysis of R\&RR clearly shows that one is limited to an accuracy of $0.8 \mathrm{mag}$ at best. Thus, $B_{3}$ can give only a first estimate of the true distance. This still excludes uncertainties which are introduced by the selection of the candidates as interlopers from the foreand background as well as unresolved blends or clusters. Furthermore, the luminosity of the BBSG's depends on the luminosity of the host galaxy. This dependance has

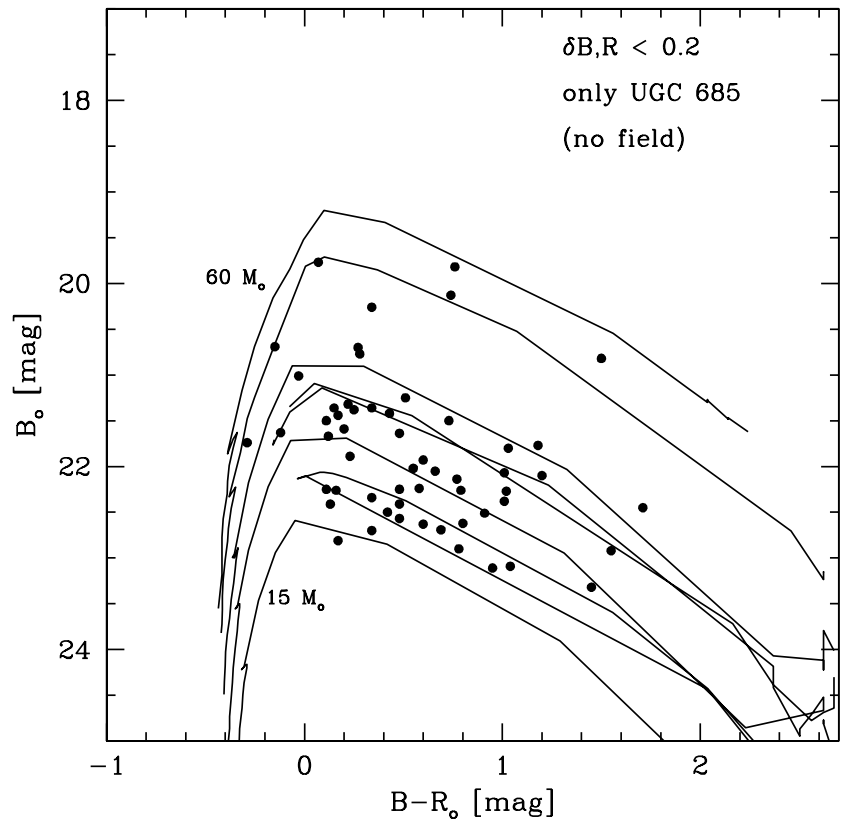

Fig. 6. $B-R, B$ color-magnitude diagram of 56 stars of UGC 685. Stars of the surrounding field and those with errors larger than 0.2 are excluded. The observations are corrected for the foreground reddening of the Milky Way. Geneva tracks for $15,20,25,40$, and $60 M_{\odot}$ stars of a metallicity of $Z=0.001$ are shown. The tracks were taken from the http version of Schaller et al. (1992) and converted from mass, temperature, and luminosity to the observers frame with Kurucz (1992) stellar atmospheres (where the tables with $\log g, T$, and colors are taken again from the web) and finally shifted to a distance of $5.5 \mathrm{Mpc}$

been explained through numerical simulations as a statistical effect (Greggio 1986, and references therein). The simulation further showed that deviations from the mean relation between $B_{3}$ and $M_{\text {gal }}$ up to \pm 1 mag. can be expected in the $B$ band for galaxies which are as faint as UGC 685. This explains the rather low accuracy of the method as derived from empirical data by R\&RR. The errors given in the following are based on the derivation by R\&RR.

I identified the four brightest candidates for supergiants (Nos. 146, 194, 217, and 250 in Table 3) where $I$ used $B-R<0.4$ as color selection criterion for blue supergiants, approximating the $B-V$ color selection of K\&T. The three brightest candidates yield a mean magnitude of $B_{3}=20.9 \pm 0.6$. To get an estimate of the uncertainty introduced by clusters or blends, I also calculated $B_{3}$ from the second to fourth brightest blue objects and got $21.4 \pm$ 0.3 . The values corrected for the Milky Way foreground extinction are 20.6 and 21.1, respectively. Following R\&RR, their relation and figure, and using the above values for $B_{3}$ and total magnitude after reddening correction, I obtain an absolute magnitude for the brightest blue supergiants of about -7.9 and a distance modules (distance) of 28.7 $(5.5 \mathrm{Mpc})$. According to R\&RR, the minimum error of a 
Table 4. Individual objects identified in $B, R$, and $J$ within UGC 685. Listed are the numbers from Table 3, magnitudes and colors, the spectral types as estimated from the color-color values, and the individual dereddened distance moduli assuming that the objects are supergiants in UGC 685. See text for details

\begin{tabular}{l|c|c|c|c|c}
\hline $\begin{array}{l}\text { star number } \\
\text { from Table 3 }\end{array}$ & $B$ & $B-R$ & $B-J$ & $\begin{array}{c}\text { Spectral } \\
\text { type }\end{array}$ & $m-M_{0}$ \\
\hline 155 & 20.54 & 0.93 & 0.66 & F1I: & 28.78 \\
176 & 22.18 & 1.36 & 1.47 & F9I & 30.12 \\
207 & 20.67 & 0.52 & 0.43 & A5I & 28.91 \\
216 & 21.33 & 1.68 & 2.18 & G5I & 28.71 \\
\hline
\end{tabular}

distance modulus derived from their relation (10f) is 0.88 . To this value, I added quadratically the error of $B_{3}$ and the - almost negligible - error in total magnitude. This yields a total error of 1.0 in distance modulus or about $35 \%$ in distance. The derived distance value was used to convert observed to absolute values as given in Table 2 and to adjust the tracks (Fig. 6). Following the calibration of $\mathrm{K} \& \mathrm{~T}$ yields almost identical values.

I was able to identify four individual sources in the $J$ frame which could be cross-correlated with the objects from the $B$ and $R$ frames (Table 4 ). The colors were corrected for the Milky Way foreground reddening. Assuming that these four objects are supergiants and that UGC 685 itself does not contribute internal reddening, one can translate the position in the $B-R_{\mathrm{c}}, B-J$ color-color diagram to a spectral type using the table of Johnson (1966). The Johnson table was transfer into Cousins $R$ with Bessels (1987) relation. Using the blue absolute magnitudes of supergiants for these spectral types as listed by Schmidt-Kaler (1982), one gets distance moduli as also given in Table 4 . Three objects combine to $m-M=28.8$ (5.8 Mpc), including the fourth one yields 29.1 (6.7 Mpc). I take this as a consistency check for the $B_{3}$ method. The deviating result for the fourth object shows the limitation of this approach and may be caused by a blend or one of the above assumptions may not be valid in this case.

The two distance estimates presented here are in rather good agreement with an estimate based on the observed HI velocity and a Virgo infall model which yields about 6.0 Mpc (Schmidt \& Boller 1992a). Thus, UGC 685 really belongs to the $10 \mathrm{Mpc}$ sample and is quite isolated in space.

\subsection{Star formation history}

According to the results described so far, UGC 685 is in the right distance for an HST multicolor study which would provide the data for a detailed reconstruction of the star formation history (Schulte-Ladbeck et al. 1998, see also the review by Grebel 1997). Even though the ground-based data presented in this paper have a much more limited resolution, they already indicate that the recent star formation rate (SFR) is rather low and that it varied only slightly during the past.
According to the results in chapter 3.2 and 3.3, the observed total $\mathrm{H} \alpha$ flux corresponds to a recent (within the last $10^{7} \mathrm{yr}$ ) SFR of $0.003 M_{\odot} \mathrm{yr}^{-1}$. As shown, this activity is spatially highly concentrated. The resolved supergiants and the underlying light distribution are related to slightly older star formation activities. The resolved supergiants are almost all inside 26 arcsec (major axis) which corresponds to $0.70 \mathrm{kpc}$ or 2 scale lengths of the overall light distribution. This indicates that averaged over a longer time interval of $\sim 10^{8} \mathrm{yr}$, the SF took place all over the central part of UGC 685 . This is supported by the absence of significant color gradients in $B-R$ and $B-J$ and the very limited deviation from the average in the color map.

A further estimator of the SFR of an actively star forming galaxy is its total blue luminosity. As described by Gallagher et al. (1984), this estimator $\alpha_{\mathrm{L}}$ is strictly valid only for a constant SFR and depends on the assumed IMF and its upper and lower mass cut-offs. It also slightly depends on the chemical composition of the system (see Gallagher et al. 1984, for complete references). The blue light is dominated by stars of $2.5 M_{\odot}>M_{*}>1 M_{\odot}$ (4 $10^{8} \mathrm{yr}>$ age $>610^{9} \mathrm{yr}$ ). Applying the Gallagher et al. formula (7) yields the same SFR as derived from the $\mathrm{H} \alpha$ flux. Thus, I have little evidence for strong variations of the SFR with time up to $\sim 10^{9} \mathrm{yr}$.

To strengthen this point, I used the total optical colors $B-R$ and $B-I$ (Table 2). I compared the observed, dereddened colors with Bruzual \& Charlot (1994) models with exponential declining star formation rate of e-folding time $\tau$. Models with $\tau \leq 5$ Gyr do not fit the colors at all and are far too red. A $\tau=10 \mathrm{Gyr}$ model can reproduce the colors rather well with deviations of less than $0.1^{3}$. Models with an exponential decay and a recent, even small, burst are in conflict with the data. Therefore, also the colors support the scenario of a simple star formation history.

In other words, the isolated dwarf irregular galaxy UGC 685 seems to have an approximately constant and low SFR, roughly stable for the past $10^{9} \mathrm{yr}$. The SFR is also quite low when compared to other galaxies of the same morphological type - actually, UGC 685 ranges at the lower end of measured SFR values of irregular galaxies. As I applied the formulae of Gallagher et al. (1984), Hunter \& Gallagher $(1985,1986)$, it is straightforward to compare the obtained SFR's of UGC 685 to the values for their sample galaxies. For dwarf irregular galaxies, they found SFR values between 0.5 and $0.0002 M_{\odot} \mathrm{yr}^{-1}$ (giant irregulars: 0.3 to 1.1 ). The SFR correlate with the absolute blue magnitude. Galaxies which have the similar $M_{B} \pm 0.5$ as UGC 685 show similar SFR values.

As indicated by the amount of detected HI gas, this galaxy can continue with star formation at its today rate for quite a long time. To do so, the reservoir of far outlying

\footnotetext{
${ }^{3}$ A formation time of $z_{\mathrm{f}}=4, h=0.6$, and $q_{0}=0.1$ are assumed; $B-J$ is not used because of some uncertainties of the NIR model colors.
} 
HI gas has to be moved into those central regions where conditions obviously support star formation. As no external trigger seems to be present, this might be a very slow process, depending on the dynamical evolution of the extended HI disk. It might even be possible that the stellar body of todays UGC 685 will already be totally dimmed to an object resembling a dwarf spheroidal before this outlying gas is able to move in. In this context, it is interesting to note that Carignan et al. (1998) found some outlying HI gas in the Sculptor dwarf spheroidal galaxy.

\section{Conclusions}

Optical broad and narrow band as well as NIR imaging data were presented and used to derive the structural parameters of the isolated dwarf galaxy UGC 685 which show very little signs of irregularities. UGC 685 is one of those dwarfs where the HI gas is reaching to far greater distances than the stars, and might be, like DDO 154, a good case for dark matter halo studies. In a separate paper, I will show that the stellar and ionized gas kinematics fit quite well to the HI rotation curve established by Hoffman et al. (1996). Total fluxes and colors, the color distribution, and the especially $\mathrm{H} \alpha$ flux and morphology point to a low star formation rate. Even for dwarf irregular galaxies, it is at the lower boundary of the observed values (Hunter \& Gallagher 1985, 1986). The overall star formation history seems to be calm at a level of $0.003 M_{\odot}$ $\mathrm{yr}^{-1}$ over the last $\sim 10^{9} \mathrm{yr}$, but this particular irregular dwarf was nevertheless able to establish a surface brightness at the upper boundary of dwarfs of this size (compare to Binggeli 1993, his Fig. 4, or Hopp 1994, Fig. 1). Over short intervals $\left(\sim 10^{7} \mathrm{yr}\right)$, the star formation activity appears strongly localized. Data with much better resolution are needed for a more detailed study and reconstruction.

A new distance estimate was established based on the resolved brightest supergiants. This new estimates confirms that UGC 685 belongs to the $10 \mathrm{Mpc}$ sample of the very nearby universe and is indeed a very isolated galaxy.

Acknowledgements. I would like to thank the Calar Alto staff for his kind support during the observations. Drs. Ralf Napiwotzki and Sabine Moehler helped with the electronic version of the Kurucz tables. I acknowledge many useful discussions with Drs. Bender, Greggio, Rosa, and Schulte-Ladbeck. Niv Drory did the Bruzual \& Charlot model calculations. I was supported by the DFG (hopp/1801-1) and by the SFB 375 .

\section{References}

Babul A., Ferguson H., 1996, ApJ 458, 100

Bender R., Möllenhoff C., 1987, A\&A 177, 71

Bessel M.S., 1987, PASP 99, 642
Bertelli G., Bressan A., Chiosi C., Fagotto F., Nasi E., 1994, A\&AS 106, 275

Binggeli B., 1993, in: "Panchromatic View of Galaxies", Hensler G., Thies Ch., Gallagher J.S. (eds.). Édition Frontières, p. 173

Bomans D., Chu Y.H., Hopp U., 1996, AJ 113, 1678

Bruzual A.G., Charlot S., 1993, ApJ 405, 538

Burstein D., Heiles C., 1982, AJ 87, 1165

Carignan C., Beaulieu S., Cote S., Demers S., Mateo M., 1998, AJ (in press) (astro-ph/9807222)

Christian C.A., Adams M., Barnes J.V., Butcher H., Mould J.R., Siegel M., 1985, PASP 97, 363

Elias J.H., Frogel J.A., Matthew K., Neugebauer G., 1982, AJ 87,1029

Ferguson H., Babul A., 1998, MNRAS (preprint, astroph/9801057)

Gallart C, Aparicio A., Vilchez J.M., 1996, AJ 112, 1928

Gallagher J.S., Hunter D.A., Tutukov A.V., 1984, ApJ 284 , 544

Grebel E.K., 1997, Rev. Modern Astron. 10, 29

Greggio L., 1986, A\&A 160, 111

Greggio L., 1994, ESO Conf. Workshop Proc. 51, 72

Hoffman G.L., Salpeter E.E., Farhat B., Roos T., Williams H., Helou G., 1996, ApJS 105, 269

Hopp U., 1994, ESO Conference \& Workshop Proc. 49, 37

Hopp U., Schulte-Ladbeck R.E., 1991, A\&A 248, 1

Hunter D., 1997, PASP 109, 937

Hunter D.A., Gallagher J.S. III, 1985, ApJS 58, 533

Hunter D.A., Gallagher J.S. III, 1986, PASP 98, 5

Hunter D.A., Hawley W.N., Gallagher J.S. III, 1994, AJ 106, 1797

Hunter D., Elmegren B.G., Backer A.L., 1998, ApJ 493, 595

Johnson H.L., 1966, ARA\&A 4, 193

Karachentsev I.D., Tikhonov N.A., 1993, ESO Conf. Workshop Proc. 49, 109 (K\&T)

Kran-Korteweg R., 1986, A\&AS 66, 255

Kran-Korteweg R., Tammann G.A., 1979, AN 300, 181

Kurucz R.L., 1992, in: The Stellar Populations of Galaxies, Barbuy B. \& Renzini A. (eds.). Dordrecht, Kluwer, p. 255

Lu N.Y., Hoffman G.L., Groff T., Roos T., Lamphier C., 1993, ApJS 88, 383

Meynet G., Maeder A., Schaller G., Schaerer D., Charbonnel C., 1994, A\&AS 103, 97

Rozanski R., Rowen-Robinson M., 1994, MNRAS 271, 530 $(\mathrm{R} \& \mathrm{RR})$

Schaller G., Schaerer D., Meynet G., Maeder A., 1992, A\&AS 96, 269

Schmidt K.H., Boller Th., 1992a, AN 313, 190

Schmidt K.H., Boller Th., 1992b, AN 313, 330

Schmidt-Kaler Th., 1982, in: Landolt-Börnstein, Numerical Data and Functional Relationships in Science and Technology, N.S. VI, 2b, Hellwege K.H. (ed.). SpringerVerlag, Berlin

Schulte-Ladbeck R.E., Crone M.M., Hopp U., 1998, ApJ 493, L23

Taylor C.L., Brinks E., Grashuis R.M., Skillman E.D., 1996 ApJS 102, 189

van Zee L., Skillman E.D., Salzer J.J., 1998, AJ (in press) (astro-ph/9806246) 